\title{
Effect of Acute Static Stretching on the Activation Patterns Using High-Density Surface Electromyography of the Gastrocnemius Muscle during Ramp-Up Task
}

\author{
Noriaki Maeda ${ }^{1, *(1)}$, Makoto Komiya ${ }^{1}\left(\mathbb{D}\right.$, Yuichi Nishikawa ${ }^{2}\left(\mathbb{D}\right.$, Masanori Morikawa ${ }^{1,3} \mathbb{D}$, Shogo Tsutsumi $^{1}$, \\ Tsubasa Tashiro ${ }^{1}{ }^{1}$, Kazuki Fukui ${ }^{1}$, Hiroaki Kimura ${ }^{4}$ and Yukio Urabe ${ }^{1}$ \\ 1 Department of Sports Rehabilitation, Graduate School of Biomedical and Health Sciences, Hiroshima \\ University, 1-2-3 Kasumi, Minami-ku, Hiroshima 734-8553, Japan; makoto-komiya@hiroshima-u.ac.jp (M.K.); \\ m-masanori@hiroshima-u.ac.jp (M.M.); shogo-tutumi@hiroshima-u.ac.jp (S.T.); \\ tsubasatashiro716@hiroshima-u.ac.jp (T.T.); kazuki-fukui@hiroshima-u.ac.jp (K.F.); \\ yurabe@hiroshima-u.ac.jp (Y.U.) \\ 2 Faculty of Frontier Engineering, Institute of Science \& Engineering, Kanazawa University, \\ Kanazawa 920-1192, Japan; yuichi@se.kanazawa-u.ac.jp \\ 3 Department of Preventive Gerontology, Center for Gerontology and Social Science, National Center for \\ Geriatrics and Gerontology, 7-430 Morioka-cho, Aichi, Obu City 474-8511, Japan \\ 4 Department of Rehabilitation, Hiroshima University Hospital, Hiroshima University, Hiroshima 734-8551, \\ Japan; luna@hiroshima-u.ac.jp \\ * Correspondence: norimmi@hiroshima-u.ac.jp; Tel.: +81-82-257-5410; Fax: +81-82-257-5344
}

\section{check for} updates

Citation: Maeda, N.; Komiya, M.; Nishikawa, Y.; Morikawa, M.; Tsutsumi, S.; Tashiro, T.; Fukui, K.; Kimura, H.; Urabe, Y. Effect of Acute Static Stretching on the Activation Patterns Using High-Density Surface Electromyography of the Gastrocnemius Muscle during Ramp-Up Task. Sensors 2021, 21, 4841. https://doi.org/10.3390/s21144841

Academic Editor: Lorenzo Chiari

Received: 14 June 2021

Accepted: 13 July 2021

Published: 15 July 2021

Publisher's Note: MDPI stays neutral with regard to jurisdictional claims in published maps and institutional affiliations.

Copyright: (c) 2021 by the authors. Licensee MDPI, Basel, Switzerland. This article is an open access article distributed under the terms and conditions of the Creative Commons Attribution (CC BY) license (https:// creativecommons.org/licenses/by/ $4.0 /)$.

\begin{abstract}
This study aimed to evaluate motor unit recruitment during submaximal voluntary ramp contraction in the medial head of the gastrocnemius muscle (MG) by high-density spatial electromyography (SEMG) before and after static stretching (SS) in healthy young adults. SS for gastrocnemius was performed in 15 healthy participants for $2 \mathrm{~min}$. Normalized peak torque by bodyweight of the plantar flexor, muscle activity at peak torque, and muscle activation patterns during ramp-up task were evaluated before and after SS. Motor unit recruitment during the submaximal voluntary contraction of the MG was measured using SEMG when performing submaximal ramp contractions during isometric ankle plantar flexion from 30 to $80 \%$ of the maximum voluntary contraction (MVC). To evaluate the changes in the potential distribution of SEMG, the root mean square (RMS), modified entropy, and coefficient of variation (CV) were calculated from the dense surface EMG data when $10 \%$ of the MVC force was applied. Muscle activation patterns during the 30 to $80 \%$ of MVC submaximal voluntary contraction tasks were significantly changed from 50 to 70\% of MVC after SS when compared to before. The variations in motor unit recruitment after SS indicate diverse motor unit recruitments and inhomogeneous muscle activities, which may adversely affect the performance of sports activities.
\end{abstract}

Keywords: static stretching; motor unit recruitment; submaximal voluntary ramp contraction; highdensity spatial electromyography

\section{Introduction}

Stretching is performed during warm-up for sports and is considered essential for optimizing performance [1,2]. It has proven to be an effective method for decreasing muscle stiffness [3], and improving joint flexibility and physical performance in activities involving jumping and dynamic balance [4]. In addition, stretching is important for the prevention of injuries during sporting activities [5]. There are several types of stretching, including static, dynamic, cyclic, and proprioceptive neuromuscular facilitation stretching; it is necessary to take into account the different effects of each stretching technique in order to identify one that would be best suited to the individual [3,4].

Static stretching (SS), which keeps the muscles stretched for a certain period of time without recoil to improve muscle flexibility, is widely used in the fields of sports and 
rehabilitation [3]. Current research suggests that SS decreases muscle activity and power and evokes contractile properties, thereby impacting physical performance [6]. A previous study reported greater activation deficits after SS than before SS [7]. These effects may have been due to peripheral nerve mechanisms resulting from muscle tendon stiffness [8]. To date, a unified view on the effects of SS is lacking.

Multichannel spatial electromyography (SEMG) was recently developed as a noninvasive technique to measure the mechanical and contractile properties of the motor unit activity of skeletal muscles using multiple electrodes arranged on a two-dimensional plane [9]. SEMG measures the number of motor units recruited by an individual during active muscle contraction [10-12]. Holtermann et al. [13] suggested that changes in the spatial distribution of SEMG could be explained by physiological changes in motor unit recruitment. These changes are underpinned by the high density of muscle fibers in a limited area and the spatial inhomogeneity of muscle fibers for certain muscles [14].

Previous studies have demonstrated that the muscle contractions induced by electrical stimulation to the muscle belly are prolonged after performing SS and induce a decrease in muscle and tendon stiffness [15]. However, to our knowledge, there is no previous study about the differences in motor unit recruitment during submaximal voluntary ramp contraction before and after SS being unclear. Therefore, this study aimed to evaluate motor unit recruitment during submaximal voluntary ramp contraction in the medial head of the gastrocnemius muscle (MG) by SEMG performed after SS compared to before SS in healthy young adults. We hypothesized that variations in motor unit recruitment would indicate diverse motor unit recruitments during ramped submaximal voluntary contraction performed after SS when compared to that before SS.

\section{Materials and Methods}

\subsection{Experimental Design}

In this study, a randomized repeated measures experimental method was used to examine the effects of SS on plantar flexion torque and MG activation patterns by performing isometric plantar flexion exercises in the range of 30 to $80 \%$ of the maximal voluntary contraction (MVC). Stretching was performed on the dominant limb, to be assessed unilaterally, in order to ensure consistency in data collection across subjects. The dominant hand was defined as the limb used to kick the soccer ball. All participants were determined to be right-leg dominant [16].

The procedure is shown in Figure 1. The warm-up consisted of a 5-min cycling exercise with the ergometer air resistance set to $75 \mathrm{~W}$ and the cadence set to $60 \mathrm{rpm}$, followed by a complete break for $3 \mathrm{~min}$ before the start of the test [17]. We then measured the normalized peak torque (NPT) using a Biodex 4 dynamometer (Sakai Medical Co., Ltd., Tokyo, Japan) and muscle activation patterns. The activation patterns of the MG were measured using SEMG when performing ramped submaximal contractions during isometric ankle plantar flexion from 30 to $80 \%$ of MVC before and immediately after SS or at 5 min after SS. Stretching was performed on the dominant leg using the Biodex 4 dynamometer in a seated position. The foot was placed at maximum dorsiflexion on the footplate using a stretching device with the Biodex 4 dynamometer while the knee joint was placed in the neutral position. The stretch was maintained for 2 min based on a former report by Kanazawa et al. [18], which stated that maximum dorsiflexion reached a plateau at $2 \mathrm{~min}$ when both the gastrocnemius and Achilles tendon underwent SS at maximum dorsiflexion in a sitting position. The degree of passive dorsiflexion was recorded using the Biodex 4 dynamometer. The maximum degree of ankle dorsiflexion was defined as the angle at which the subjects began to experience pain. 


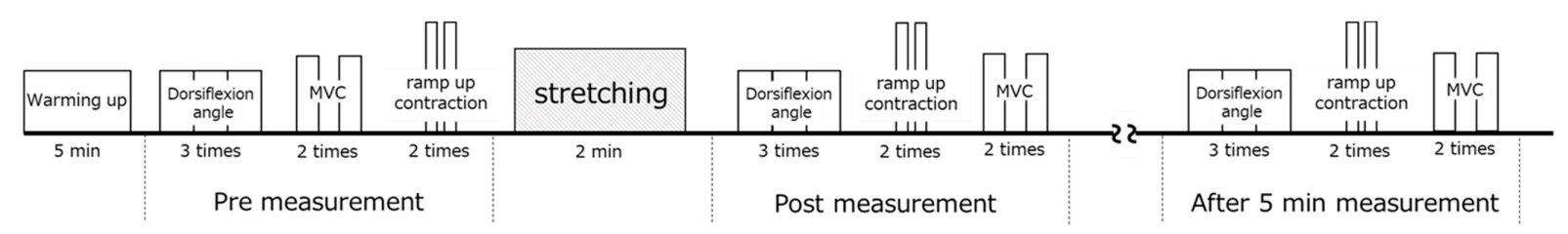

Figure 1. Flowchart of the measurement procedure. MVC: maximal voluntary contraction.

\subsection{Participants}

In total, 16 healthy male recreationally active subjects (mean age \pm standard deviation [SD], $24.7 \pm 2.4$ years; height, $170.7 \pm 6.1 \mathrm{~cm}$; body mass, $63.2 \pm 8.2 \mathrm{~kg}$ ) voluntarily participated in this study. "Recreationally active" was defined as participating in at least one exercise session per week in the past two months and not participating in any structured exercise training during this period. We excluded participants with injuries in the lower extremities and/or neuromuscular disease. Subjects performed both conditions in various orders in a two-day period with intervals of no less than $24 \mathrm{~h}$ and no more than $48 \mathrm{~h}$ between tests. All of the subjects were able to complete the task. Three successful trials were conducted in each condition and were used for data analysis. The average duration of trials in each laboratory was $50.0 \pm 4.0 \mathrm{~min}$.

All subjects provided informed consent to participate in the study. The study protocol met the requirements of the Declaration of Helsinki and was approved by the Ethical Committee for Epidemiology of Hiroshima University (approval number E-2006).

\subsection{Assessment of Normalized Peak Torque of Plantar Flexor}

In order to assess the peak torque of the plantar flexor muscle, participants were instructed to plantar flex their ankle gradually by maximally contracting the muscle for 0 to $5 \mathrm{~s}$ and maintaining it for 2 of the $5 \mathrm{~s}$. Subjects performed at least two MVC trials with $>120$ s rest between trials after one or two practice sessions [19].

\subsection{Assessment of SEMG}

After NPT, subjects were instructed to perform a forceful ramp contraction from 0 to $80 \%$ of MVC with a rate of increase of approximately $10 \%$ of the MVC per second [20]. Subjects were presented with the target torque generated on the monitor of a personal computer and practiced performing MVC and ramp submaximal contractions for $>10 \mathrm{~min}$ before the session.

\subsection{SEMG Recording}

The SEMG signals of MG were recorded using a 64-electrode semi-disposable grid (ELSCH064RS3, OT Bioelettronica, Torino, Italy) using the same procedure as that used in previous studies [20]. The grid consisted of five rows and 13 columns of electrodes (1-mm diameter, 8-mm distance between electrodes), with one missing electrode in the upper left corner (Figure 2). Before attaching the electrodes to the skin with an adhesive sheet (KITAD064, OT Bioelettronica), the calf hair was removed, the skin was cleaned with alcohol, and a conductive paste (Elefix Z-181BE, NIHON KOHDEN, Tokyo, Japan) corresponding to the electrode placement was applied.

The SEMG electrode grid was placed on the MG. The center of the electrode grid was attached to the MG near the point of the maximal cross-sectional area of the lower leg, which was at a level of $30 \%$ of the lower leg length from the medial popliteal fossa to the calcaneal insertion (Figure 2). Before attaching the electrode sheet, ultrasound measurements were performed underneath to confirm whether the MG was included in the area of the electrode sheet. The rows of the electrode grid were placed parallel to the longitudinal axis of the MG, and the site of the missing electrode was proximal to the MG based on a previous study [20]. Thereafter, the electrode grid was attached to the computer through a 12-bit analog-todigital converter (EMG-USB2+, OT Bioelettronica). The recording of the SEMG signals was 
controlled using a software program (OTBioLab+ v1.4.2.0, Bioelettronica, Torino, Italy). Two reference electrodes were attached at the fibular head and the center of the patella, respectively. The same investigator performed all procedures. The SEMG monopole signal was amplified by a factor of 1000, sampled at $2048 \mathrm{~Hz}$ per channel, and converted to digital data using a 12-bit analog-to-digital converter. The recorded monopolar signals were bandpass-filtered (10-500 Hz) off-line and analyzed using software (MATLAB 2018a, MathWorks GK, Massachusetts, USA). Bipolar multichannel SEMG signals $(n=59)$ along the column were segmented from 64 electrodes; SEMG signals were sampled between $3 \mathrm{~s}$ ( $30 \%$ of MVC) and $8 \mathrm{~s}(80 \%$ of MVC) of the ramp-up protocol, and the root mean square (RMS) was calculated. The RMS estimates were normalized by the value of MVC; the coefficient of variation (CV) of force (SD/mean $\mathrm{x} 100, \mathrm{CV}$ force) was calculated in the same time series as the coefficient of variation of SEMG variables [20]. Modified Entropy, RMS, and $\mathrm{CV}$ were determined to characterize the heterogeneity of the potential distribution of SEMG at each contraction time. The modified entropy was obtained over a $1 \mathrm{~s}$ epoch obtained during a tilted submaximal contraction, and this value was calculated by the following equation after calculating 59 RMS values (in space) of a single difference signal from the EMG amplitudes of muscle activity in a wide range of muscles [11]:

$$
E=-\sum_{i=1}^{59} p(i)^{2} \log _{2} p(i)^{2}
$$

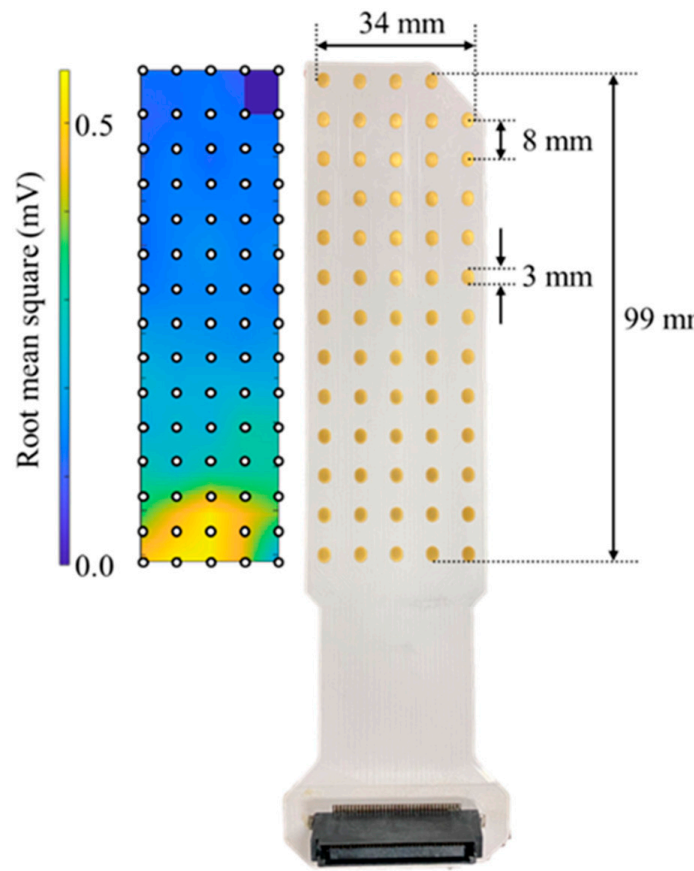

A

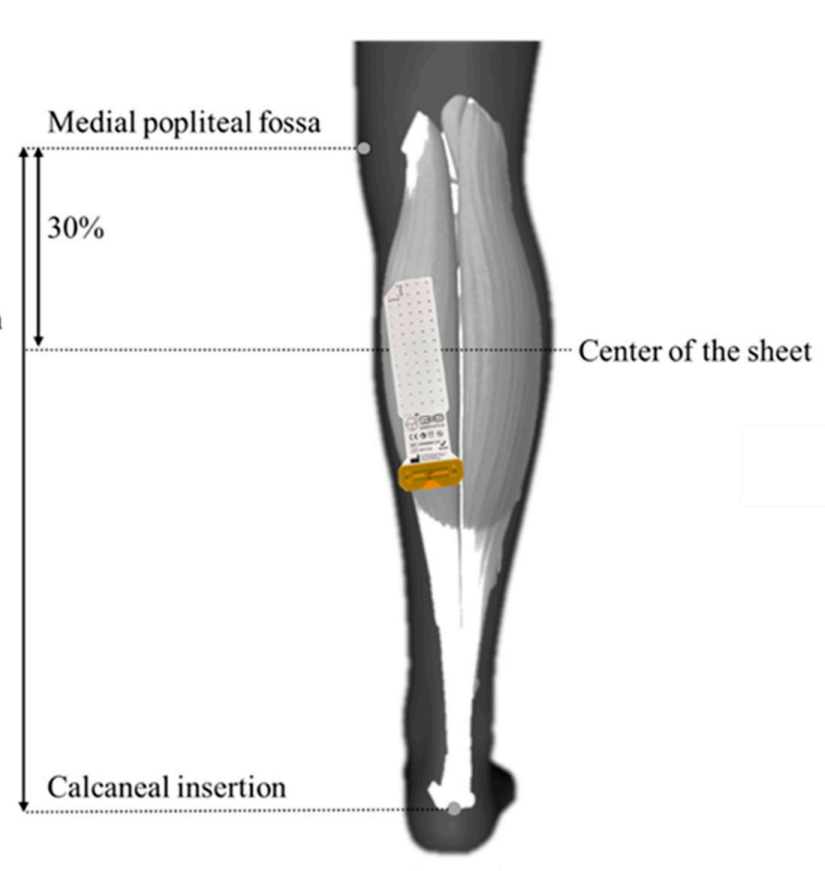

B

Figure 2. The location of surface electromyography application and amplitude values of electrodes. (A): A color map of the typical spatial distribution pattern of the amplitude values of a surface electromyogram. The grid consists of 13 rows and five columns. In the present study, one site of the proximal part of the MG muscle (upper right part of the electrode) was missing. (B): The electrode grid of the multichannel electromyography was placed at the medial head of the gastrocnemius muscle. The central part of the electrode grid was attached to the center of the line connecting the medial knee fossa and calcaneal insertion. Reference electrodes were attached at the position of the fibular head and patella.

The $\mathrm{p}(\mathrm{i})$ in the above equation is the square of the RMS value of channel i divided by the sum of the squares of the 59 RMS values at a given contraction time. Thus, the normalized power of each channel was expressed as $p(i)^{2}$. The CV of RMS was defined as the mean value of the 59 RMS measurements at each time point and a quarter of the 
SD of the 59 RMS measurements. We calculated the change in two variables (modified entropy and CV of RMS) to examine the change in the recruitment of motor units over time. These variables were calculated between contraction times of $2 \mathrm{~s}$ and $8 \mathrm{~s}$. The decrease in the modified entropy and the increase in the CV of RMS indicated an increase in the heterogeneity of the SEMG potential distribution in the electrode grid [21]. Recent studies have quantified the spatial distribution pattern of SEMG and used SEMG to estimate the recruitment pattern of motor units [22].

\subsection{Statistical Analysis}

Data were analyzed using EZR (Saitama Medical Centre, Jichi Medical University, Saitama, Japan) [23]. Continuous data are presented as the mean \pm SD or median (min, max). Before the analysis, data normality was confirmed using the Shapiro-Wilk test. A one-factor repeated measure ANOVA was conducted to compare NPT, RMS, modified entropy, and CV among the conditions (pre, post, and post- $5 \mathrm{~min}$ ) if the normality was confirmed. A Friedman test was conducted if the abnormality was confirmed for each condition (pre, post, and post- $5 \mathrm{~min}$ ). Differences between torque levels were analyzed using the Holm post hoc test to adjust the $\mathrm{p}$ value for multiple comparisons. A significance level of $p<0.05$ was used.

\section{Results}

The color map of representative multichannel SEMG amplitudes is shown in Figure 3. Differences in the potential distribution of SEMG at each contraction time pre- and post-SS were observed. Significant differences were not noted between NPT immediately after SS and NPT at 5 min after SS (Figure 4).

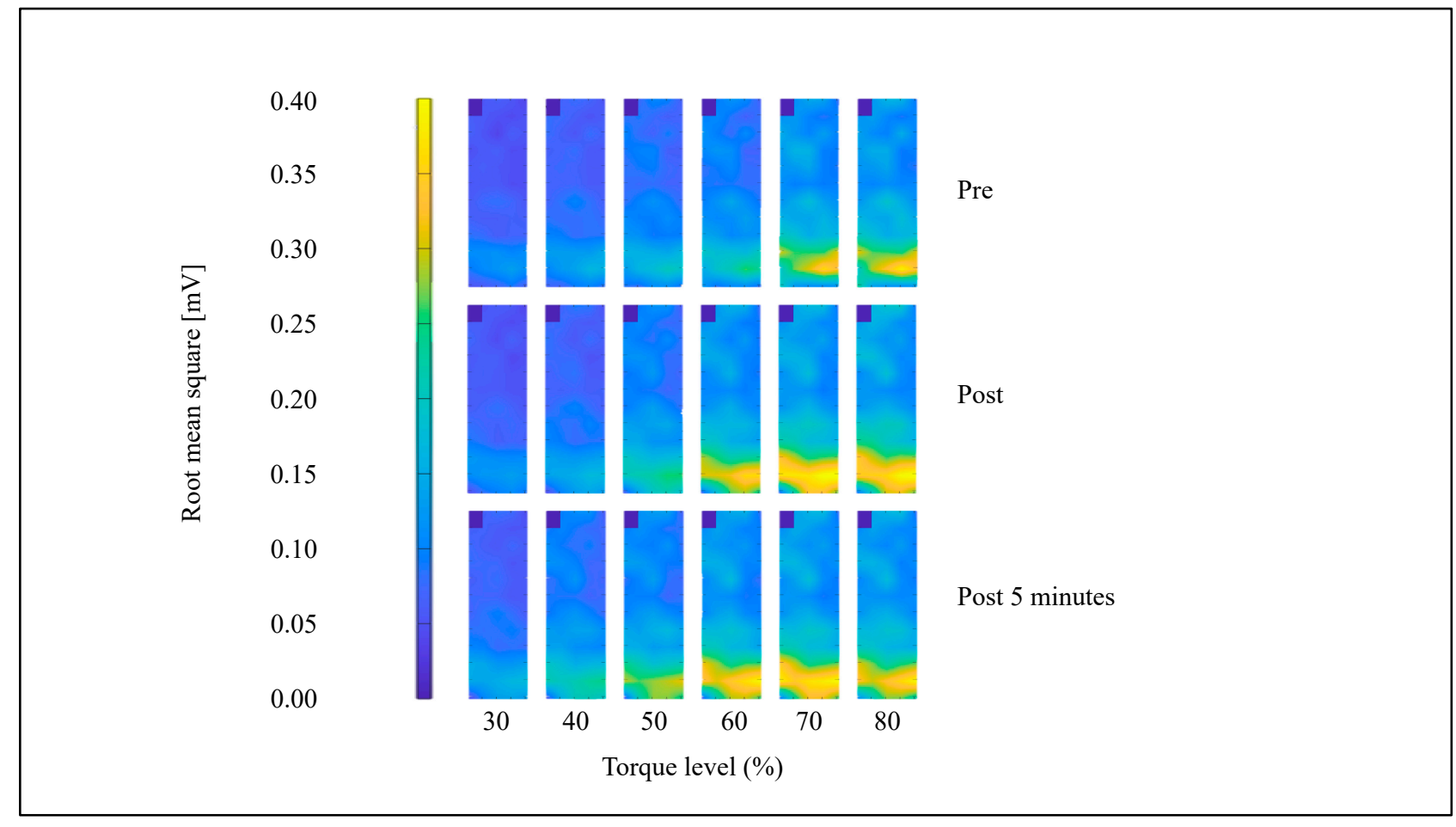

Figure 3. Root mean square of multichannel surface electromyogram amplitudes at 30 to $80 \%$ of the maximum voluntary contraction between conditions. 


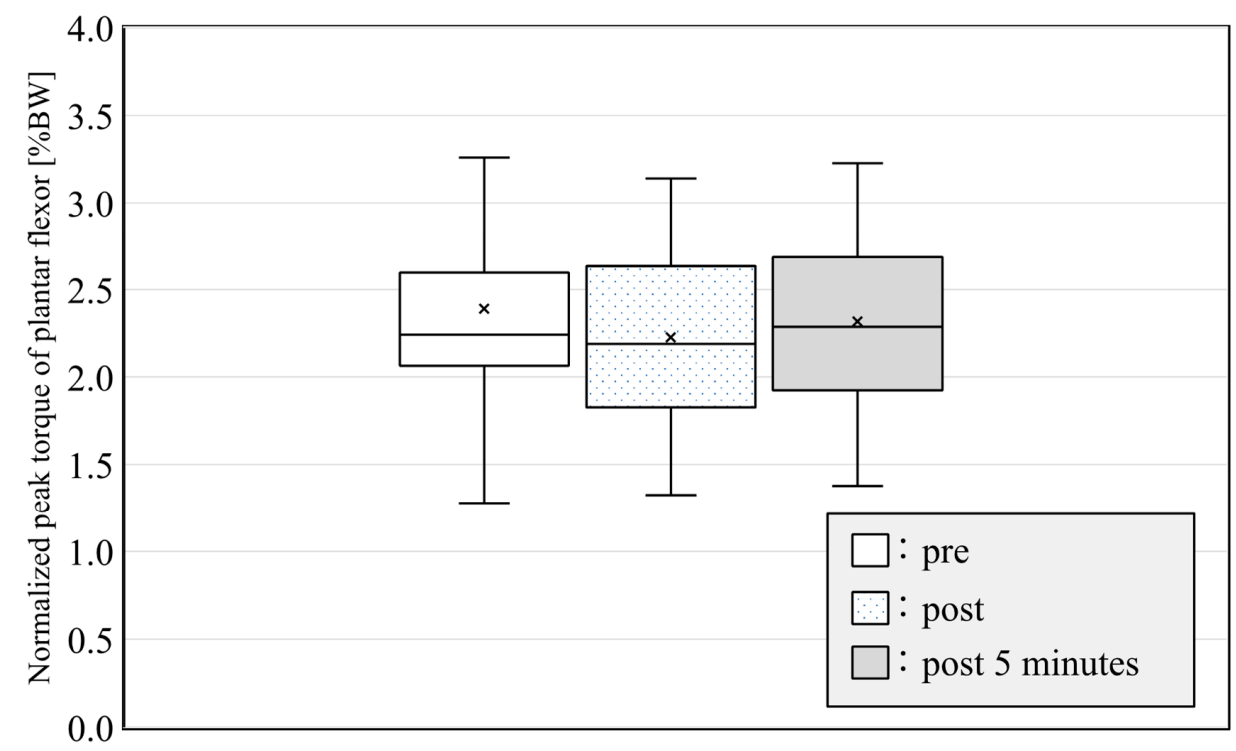

Figure 4. Normalized peak torque of the plantar flexor at pre, post, and post-5-min conditions.

All subjects were able to perform the submaximal ramp contraction from 10 to $80 \%$ of MVC. RMS after SS was significantly increased from 50 to $80 \%$ of MVC immediately after SS and from 50 to $60 \%$ of MVC at 5 min after SS (Figure 5, $p<0.05$ ) in the MG. Significant differences were observed in the modified entropy and CV between pre-, immediately post-, and 5-min post-SS. The modified entropy of RMS from 40 to $60 \%$ of MVC immediately after SS and from 30 to $60 \%$ of MVC at 5 min after SS was significantly lower than that before SS (Figure $6, p<0.05$ ). In contrast, the CV of RMS from 30 to $60 \%$ of MVC immediately after SS and from 30 to $70 \%$ of MVC at 5 min after SS was significantly higher than that before SS (Figure $7, p<0.05$ ).

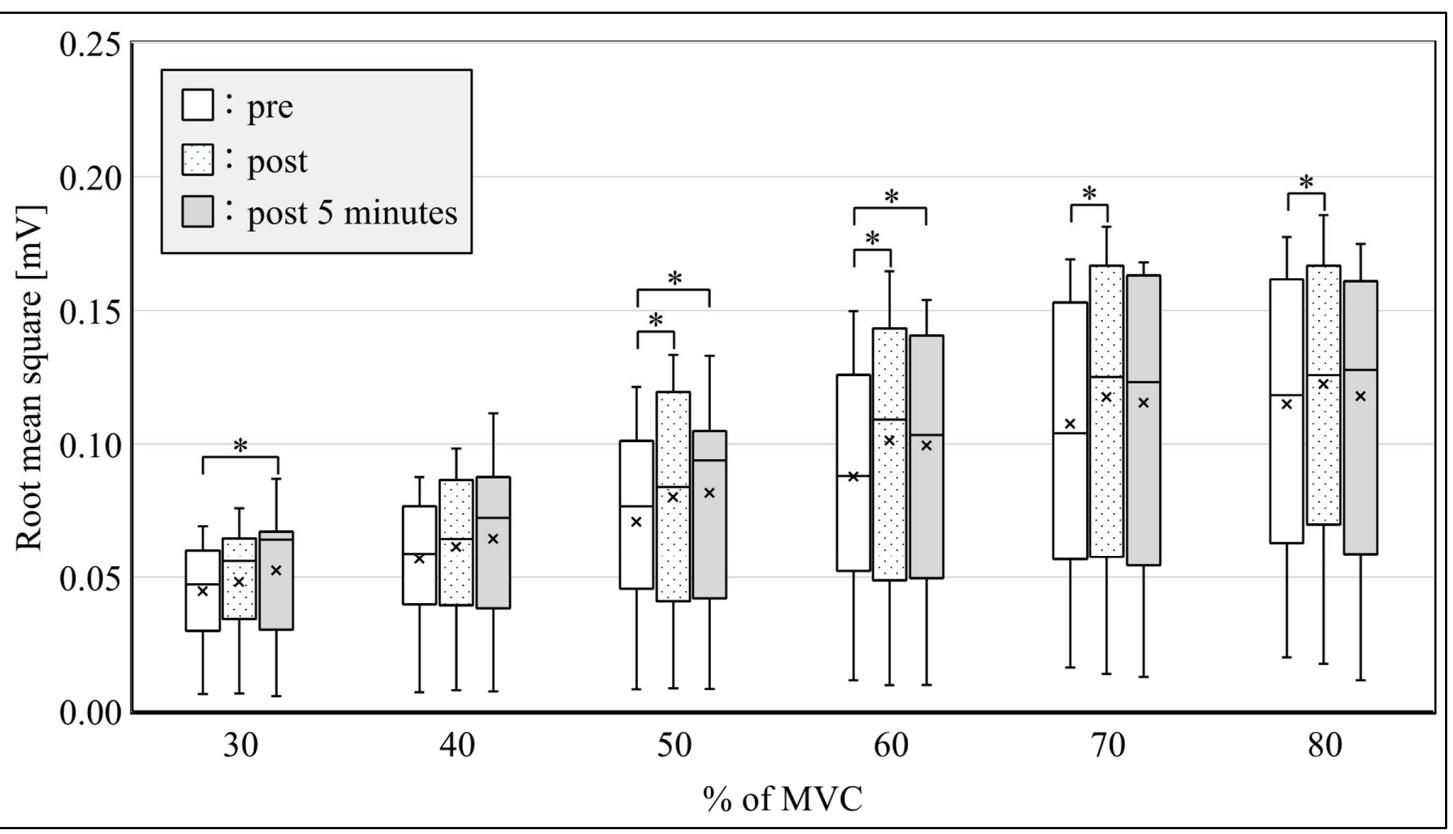

Figure 5. Root mean square of multichannel surface electromyogram amplitudes during ramp contractions at pre, post, and post-5-min conditions. ${ }^{*} p<0.05$. MVC: maximal voluntary contraction. 


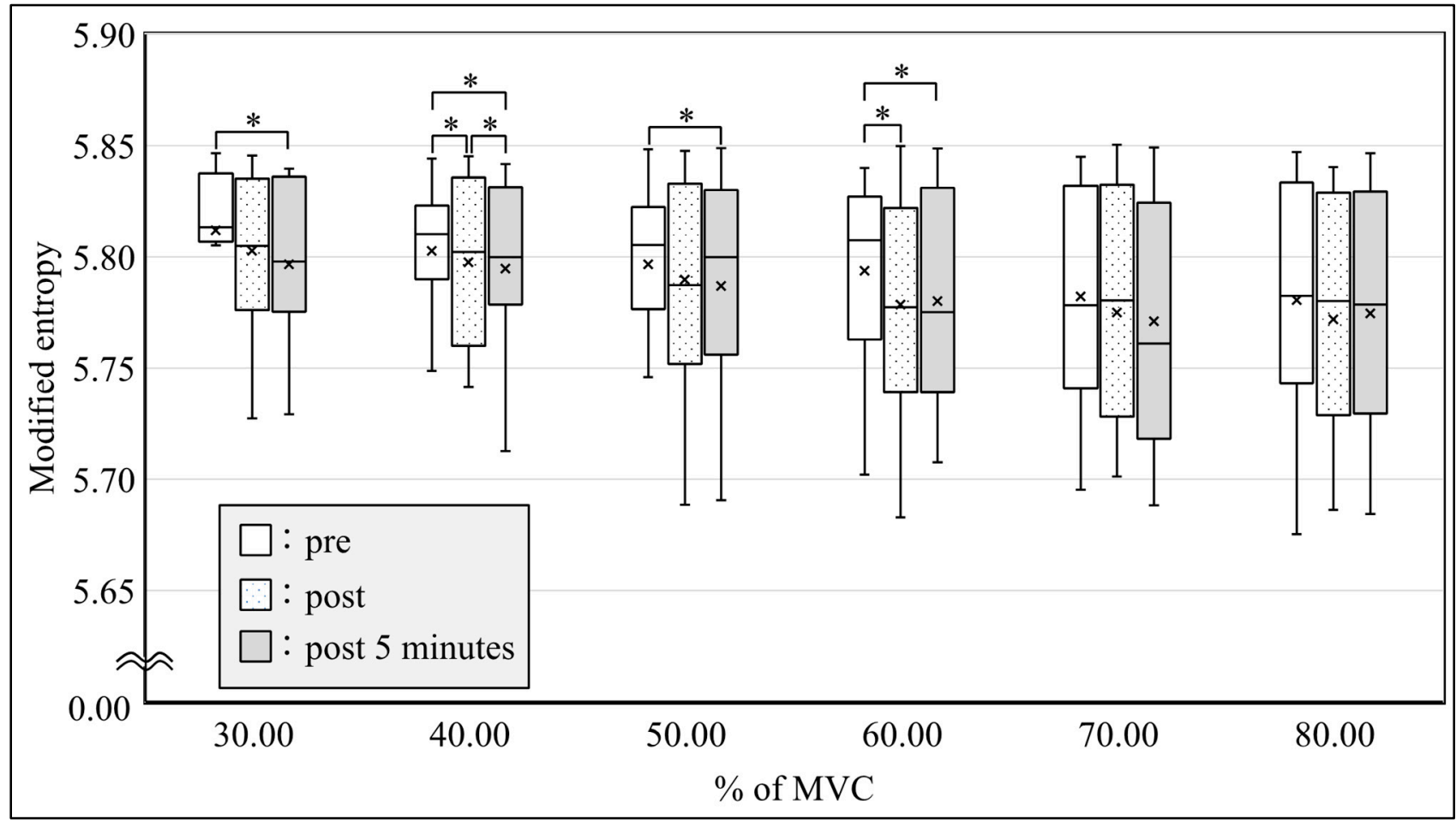

Figure 6. Comparison of the modified entropy in the amplitude of the multichannel surface electromyography during ramp contractions between pre, post, and post-5-min conditions. ${ }^{*} p<0.05$. MVC: maximal voluntary contraction.

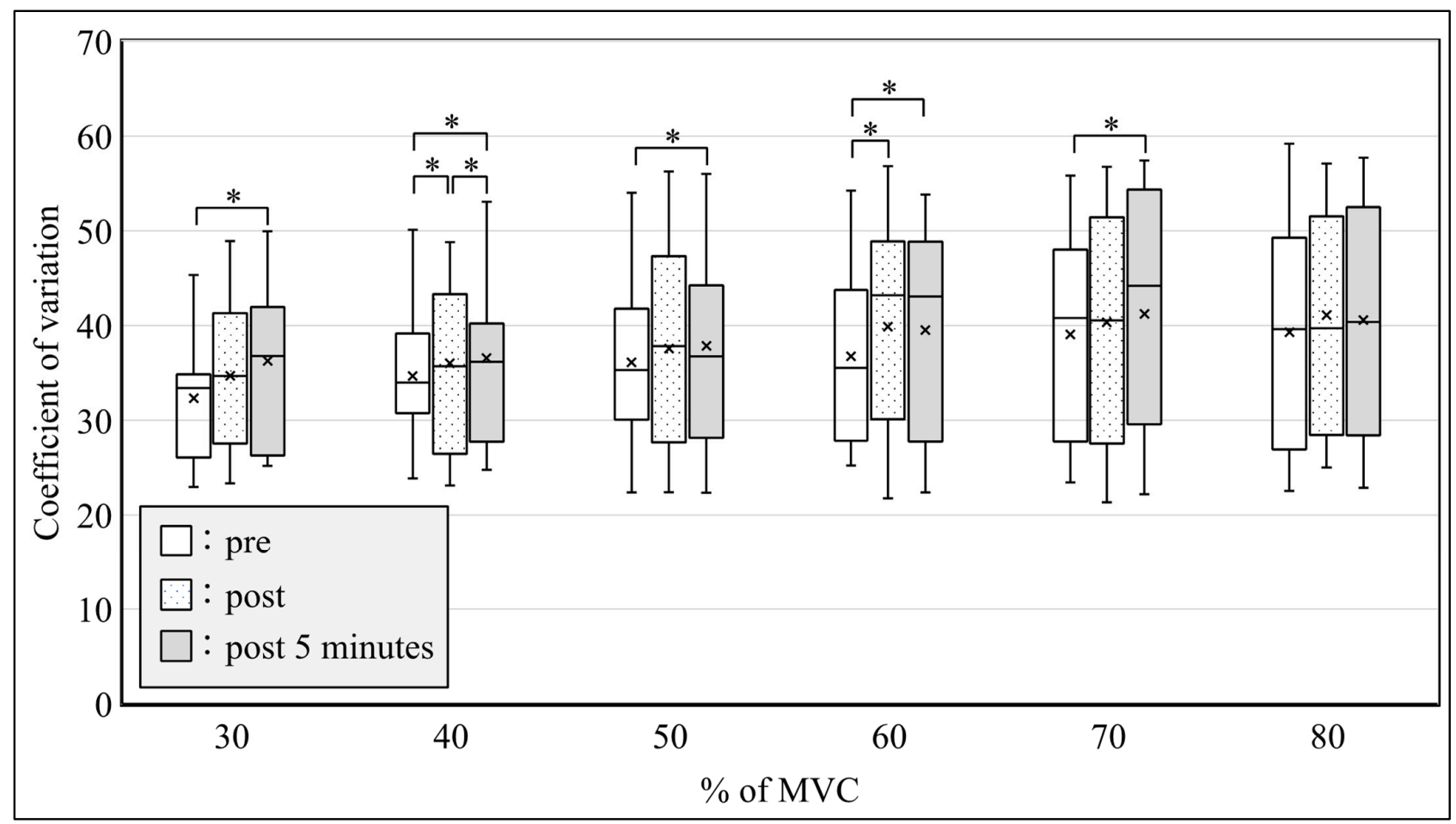

Figure 7. Coefficient of variation in the amplitude of the multichannel surface electromyography during ramp contractions at pre, post, and post-5-min conditions. ${ }^{*} p<0.05$. MVC: maximal voluntary contraction. 


\section{Discussion}

This study evaluated, in healthy young adults, the motor unit recruitment during submaximal voluntary ramp contraction in MG by an SEMG performed after SS compared to one performed before it. The primary results of the present study were as follows: the acute effects on the motor unit recruitment during ramped submaximal voluntary contraction after SS exhibited (1) significantly lower NPT and (2) significant changes in RMS, modified entropy, and CV after SS compared to before SS. These findings supported our hypothesis that variations in motor unit recruitment indicated diverse motor unit recruitments during ramped submaximal voluntary contractions performed after SS relative to those performed before it.

NPT of the plantar flexors was significantly lower immediately after and at $5 \mathrm{~min}$ after SS when compared to before it. Studies have reported decreased muscle power or evoked contractile properties after SS in the acute phase [9,24-26], which is in accordance with our findings. For these reasons, peripheral nerve mechanisms (more specifically, reduced muscle tendon stiffness) caused muscle weakness immediately after SS [26-30]. However, Magunusson et al. [31] reported that continued stretching of the muscle-tendon complex decreased the static torque. Therefore, it is necessary to analyze whether the type, intensity, frequency, and duration of stretching of the muscle-tendon complex reduces muscle exertion.

All subjects completed the ramp-up contraction task. The RMS of 50 to $80 \%$ of MVC immediately after SS and the RMS of 50 to $60 \%$ of MVC at 5 min after SS exhibited a greater force fluctuation in the ramp-up contraction task than that before SS. In this study, the muscle force fluctuation after SS required a significantly greater activation of MG than that before SS, which may have influenced the accuracy of the muscle force before SS to match the required force level.

These results indicated that the motor unit recruitment immediately after SS and at 5 min after SS involved diverse motor unit recruitments and inhomogeneous muscle activation patterns during the contraction task compared to that before SS. Watanabe et al. [21] reported that the values of modified entropy and CV indicated the heterogeneity of the spatial multichannel SEMG potential distribution within the electrode grid. Spatially distinct and changing functional properties are expected during muscle contraction, and spatially nonuniform SEMG signals are likely to be noted within the individual muscles [32]. Moreover, Farina et al. [11] suggested that the heterogeneity during a static contraction was related to the endurance time of muscles and was in line with the effect of SS for muscle conditioning. The potential for changes in the SEMG distribution during ramped submaximal contraction after SS may be related to peripheral changes, such as size changes in the motor unit area, location, type, distribution, and central nervous system adaptation. Generally, motor units are recruited to exert less force to enable muscles to contract at lower forces, and larger muscles are recruited when higher forces are required with ramp-up contraction tasks. Abnormalities may arise from reductions in musculotendinous stiffness $[8,27,29]$. Although our findings may only partially encapsulate the physiological changes after SS, they provide pertinent insights into the changes in the motor unit recruitment and inhomogeneous muscle activation patterns during ramped submaximal contraction performed after SS. SS can cause sagging by inducing stretching of the musculotendinous unit (MTU), resulting in increased electromechanical delays that affect force transfer and may reduce elastic energy [33]. In addition, Power et al. [34] also found that SS increased tendon compliance, thereby reducing force production.

Several limitations of the study need to be considered. The SEMG amplitude estimates combine properties in the peripheral and central motor units and only provide a crude estimate of motor unit recruitment [35]. Further research that utilizes analytical methods is needed to elucidate the mechanisms underscoring the inhomogeneous muscle activation patterns before and after SS. Second, we only assessed MVC and muscle activation patterns during ramped submaximal voluntary contractions, but not those of physical performances such as jump height. Further studies are needed to investigate the effects after SS on other 
muscle activation patterns and physical performance. Third, the possibility of changes in the SEMG distribution during tilted submaximal contraction after SS is thought to be related to peripheral changes, such as size changes in the area, location, type, and distribution of motor units and adaptations of the central nervous system, so this is unclear until the same changes occur in muscles other than MG.

\section{Conclusions}

In conclusion, our findings demonstrate that variations in motor unit recruitment indicate diverse motor unit recruitments and inhomogeneous muscle activation patterns during ramped submaximal contractions performed after SS. Moreover, SS can negatively affect the muscle activation patterns during submaximal ramp contractions that are performed after it, which may affect movement during sports activities after SS. Further research is needed in order to understand the underlying mechanisms.

Author Contributions: Conceptualization, N.M. and M.K.; methodology, N.M. and Y.N.; software, M.K. and S.T.; validation, S.T. and K.F.; formal analysis, M.M. and K.F.; resources, Y.N. and H.K.; data curation, T.T.; writing — original draft preparation, writing—review and editing, N.M., M.K. and M.M.; visualization, Y.U.; supervision, Y.U.; project administration, N.M. and Y.U. All authors have read and agreed to the published version of the manuscript.

Funding: This research received no external funding.

Institutional Review Board Statement: This study was authorized by the Institutional Review Board at Hiroshima university (ID number: E-2006).

Informed Consent Statement: Written informed consent was obtained from all subjects involved in the study.

Data Availability Statement: Not applicable.

Conflicts of Interest: The authors declare no conflict of interest.

\section{References}

1. Bishop, D. Warm up I: Potential mechanisms and the effects of passive warm up on exercise performance. Sports Med. 2003, 33, 439-454. [CrossRef]

2. Lewis, J. A systematic literature review of the relationship between stretching and athletic injury prevention. Orthop. Nurs. 2014, 33, 312-320. [CrossRef] [PubMed]

3. Maeda, N.; Urabe, Y.; Tsutsumi, S.; Sakai, S.; Fujishita, H.; Kobayashi, T.; Asaeda, M.; Hirata, K.; Mikami, Y.; Kimura, H. The Acute Effects of Static and Cyclic Stretching on Muscle Stiffness and Hardness of Medial Gastrocnemius Muscle. J. Sports. Sci. Med. 2017, 16, 514-520.

4. Maeda, N.; Urabe, Y.; Fujii, E.; Moriyama, N.; Iwata, S.; Sasadai, J. The effect of different stretching techniques on ankle joint range of motion and dynamic postural stability after landing. J. Sports Med. Phys. Fitness 2016, 56, 692-698.

5. Zakaria, A.A.; Kiningham, R.B.; Sen, A. Effects of Static and Dynamic Stretching on Injury Prevention in High School Soccer Athletes: A Randomized Trial. J. Sport. Rehabil. 2015, 24, 229-235. [CrossRef]

6. Behm, D.G.; Blazevich, A.J.; Kay, A.D.; McHugh, M. Acute effects of muscle stretching on physical performance, range of motion, and injury incidence in healthy active individuals: A systematic review. Appl. Physiol. Nutr. Metab. 2016, 41, 1-11. [CrossRef]

7. Kay, A.D.; Blazevich, A.J. Isometric contractions reduce plantar flexor moment, Achilles tendon stiffness, and neuromuscular activity but remove the subsequent effects of stretch. J. Appl. Physiol. 2009, 107, 1181-1189. [CrossRef] [PubMed]

8. Opplert, J.; Genty, J.B.; Babault, N. Do Stretch Durations Affect Muscle Mechanical and Neurophysiological Properties? Int. J. Sports Med. 2016, 37, 673-679. [CrossRef] [PubMed]

9. Nishikawa, Y.; Watanabe, K.; Takahashi, T.; Maeda, N.; Maruyama, H.; Kimura, H. The effect of electrical muscle stimulation on quadriceps muscle strength and activation patterns in healthy young adults. Eur. J. Sport. Sci. 2020, 1-9. [CrossRef]

10. Farina, D.; Leclerc, F.; Arendt-Nielsen, L.; Buttelli, O.; Madeleine, P. The change in spatial distribution of upper trapezius muscle activity is correlated to contraction duration. J. Electromyogr. Kinesiol. 2008, 18, 16-25. [CrossRef]

11. Holtermann, A.; Grönlund, C.; Stefan, K.J.; Roeleveld, K. Spatial distribution of active muscle fibre characteristics in the upper trapezius muscle and its dependency on contraction level and duration. J. Electromyogr. Kinesiol. 2008, 18, 372-381. [CrossRef] [PubMed]

12. Merletti, R.; Holobar, A.; Farina, D. Analysis of motor units with high-density surface electromyography. J. Electromyogr. Kinesiol. 2008, 18, 879-890. [CrossRef] [PubMed] 
13. Holtermann, A.; Roeleveld, K.; Mork, P.J.; Grönlund, C.; Karlsson, J.S.; Andersen, L.L.; Olsen, H.B.; Zebis, M.K.; Sjøgaard, G.; Søgaard, K. Selective activation of neuromuscular compartments within the human trapezius muscle. J. Electromyogr. Kinesiol. 2009, 19, 896-902. [CrossRef] [PubMed]

14. Chanaud, C.M.; Macpherson, J.M. Functionally complex muscles of the cat hindlimb. III. Differential activation within biceps femoris during postural perturbations. Exp. Brain Res. 1991, 85, 271-280. [CrossRef]

15. Esposito, F.; Limonta, E.; Cè, E. Passive stretching effects on electromechanical delay and time course of recovery in human skeletal muscle: New insights from an electromyographic and mechanomyographic combined approach. Eur. J. Appl. Physiol. 2011, 111, 485-495. [CrossRef] [PubMed]

16. Hicks, K.M.; Onambélé, G.L.; Winwood, K.; Morse, C.I. Muscle Damage following Maximal Eccentric Knee Extensions in Males and Females. PLoS ONE 2016, 11, e0150848. [CrossRef] [PubMed]

17. Girard, O.; Bishop, D.J.; Racinais, S. M-wave normalization of EMG signal to investigate heat stress and fatigue. J. Sci. Med. Sport 2018, 21, 518-524. [CrossRef] [PubMed]

18. Kanazawa, H.; Urabe, Y.; Iwamoto, H.; Shirakawa, T. Determining the effective stretching time for achilles tendon extension. Jpn. J. Clin. Sports Med. 2009, 17, 13-17.

19. Kharazi, M.; Bohm, S.; Theodorakis, C.; Mersmann, F.; Arampatzis, A. Quantifying mechanical loading and elastic strain energy of the human Achilles tendon during walking and running. Sci. Rep. 2021, 11, 1-13. [CrossRef]

20. Nishikawa, Y.; Watanabe, K.; Takahashi, T.; Orita, N.; Kimura, H.; Matsumoto, M.; Maruyama, H. Spatial electromyography distribution pattern of the vastus lateralis muscle during ramp up contractions in Parkinson's disease patients. J. Electromyogr. Kinesiol. 2017, 37, 125-131. [CrossRef]

21. Watanabe, K.; Kouzaki, M.; Merletti, R.; Fujibayashi, M.; Moritani, T. Spatial EMG potential distribution pattern of vastus lateralis muscle during isometric knee extension in young and elderly men. J. Electromyogr. Kinesiol. 2012, 22, 74-79. [CrossRef]

22. Watanabe, K.; Kouzaki, M.; Moritani, T. Spatial EMG potential distribution of biceps brachii muscle during resistance training and detraining. Eur. J. Appl. Physiol. 2015, 115, 2661-2670. [CrossRef] [PubMed]

23. Kanda, Y. Investigation of the freely available easy-to-use software 'EZR' for medical statistics. Bone Marrow Transplant. 2013, 48, 452-458. [CrossRef] [PubMed]

24. Siatras, T.A.; Mittas, V.P.; Mameletzi, D.N.; Vamvakoudis, E.A. The duration of the inhibitory effects with static stretching on quadriceps peak torque production. J. Strength Cond. Res. 2008, 22, 40-46. [CrossRef] [PubMed]

25. Winchester, J.B.; Nelson, A.G.; Kokkonen, J. A single 30-s stretch is sufficient to inhibit maximal voluntary strength. Res. Q. Exerc. Sport. 2009, 80, 257-261. [CrossRef] [PubMed]

26. Opplert, J.; Babault, N. Acute Effects of Dynamic Stretching on Muscle Flexibility and Performance: An Analysis of the Current Literature. Sports Med. 2018, 48, 299-325. [CrossRef] [PubMed]

27. Herda, T.J.; Costa, P.B.; Walter, A.A.; Ryan, E.D.; Cramer, J.T. The time course of the effects of constant-angle and constant-torque stretching on the muscle-tendon unit. Scand. J. Med. Sci. Sports 2014, 24, 62-67. [CrossRef]

28. Matsuo, S.; Suzuki, S.; Iwata, M.; Banno, Y.; Asai, Y.; Tsuchida, W.; Inoue, T. Acute effects of different stretching durations on passive torque, mobility, and isometric muscle force. J. Strength Cond. Res. 2013, 27, 3367-3376. [CrossRef]

29. Mizuno, T.; Matsumoto, M.; Umemura, Y. Viscoelasticity of the muscle-tendon unit is returned more rapidly than range of motion after stretching. Scand. J. Med. Sci. Sports 2013, 23, 23-30. [CrossRef]

30. Cè, E.; Longo, S.; Rampichini, S.; Devoto, M.; Limonta, E.; Venturelli, M.; Esposito, F. Stretch-induced changes in tension generation process and stiffness are not accompanied by alterations in muscle architecture of the middle and distal portions of the two gastrocnemii. J. Electromyogr. Kinesiol. 2015, 25, 469-478. [CrossRef]

31. Magnusson, S.P.; Aagaard, P.; Larsson, B.; Kjaer, M. Passive energy absorption by human muscle-tendon unit is unaffected by increase in intramuscular temperature. J. Appl. Physiol. 2000, 88, 1215-1220. [CrossRef] [PubMed]

32. Anders, C.; Patenge, S.; Sander, K.; Layher, F.; Biedermann, U.; Kinne, R.W. Detailed spatial characterization of superficial hip muscle activation during walking: A multi-electrode surface EMG investigation of the gluteal region in healthy older adults. PLoS ONE 2017, 12, e0178957. [CrossRef] [PubMed]

33. Nelson, A.G.; Driscoll, N.M.; Landin, D.K.; Young, M.A.; Schexnayder, I.C. Acute effects of passive muscle stretching on sprint performance. J. Sports Sci. 2005, 23, 449-454. [CrossRef]

34. Power, K.; Behm, D.; Cahill, F.; Carroll, M.; Young, W. An acute bout of static stretching: Effects on force and jumping performance. Med. Sci. Sports Exerc. 2004, 36, 1389-1396. [CrossRef]

35. Farina, D.; Merletti, R.; Enoka, R.M. The extraction of neural strategies from the surface EMG: An update. J. Appl. Physiol. 2014, 117, 1215-1230. [CrossRef] [PubMed] 